\title{
THE EFFECTS OF TOPICAL PEPPERMINT AROMATHERAPY ON REDUCING UREMIC PRURITUS IN CHRONIC KIDNEY DISEASE PATIENTS UNDERGOING HEMODIALYSIS
}

\author{
Friska Sembiring*, Siti Saidah Nasution, Yesi Ariani \\ Faculty of Nursing, Universitas Sumatera Utara, Indonesia
}

\section{Article Information}

Received: 12 January 2019

Revised: 6 October 2020

Accepted: 24 March 2021

\section{*Corresponding Author}

Friska Sembiring

friskasembiring09@gmail.com

\section{DOI}

10.20884/1.jks.2021.16.1.1273

\begin{abstract}
Uremic pruritus is one of the chronic renal failure patients' complaints, which is an uncomfortable and itchy sensation with multifactorial causes. Peppermint complementary therapy is an essential oil with the main component of mentho $(50-60 \%)$, which provides a cold sensation to the skin. The instrument used in this quasi-experimental study was demographic data and the 5-D itch scale questionnaire. The total sample was 98 participants. The intervention was carried out by administering topical peppermint essential oil. The results showed that the uremic pruritus scale decreased to a mild degree of $51.0 \%$. After being tested by Wilcoxon and Mann Whitney statistical tests, the intervention group had a $p$ value of $0.000<p=0.05$ and in the control group there was a $p$ value of 0.102 so there was an effect of giving peppermint aromatherapy in reducing uremic pruritus. Peppermint aromatherapy is safe to use and is easy to obtain so therefore it can be applied topically to improve coping strategies for patients who experience uremic pruritus.
\end{abstract}

Keywords: Aromatherapy; uremic pruritus; chronic kidney failure; hemodialysis

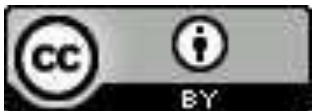

ISSN : 1907-6637

e-ISSN : 2579-9320

\section{INTRODUCTION}

Kidney failure occurs when the kidneys cannot remove metabolic waste from the body or carry out their regulatory functions. One of the types of kidney failure is chronic kidney disease where the kidney function is damaged for years. This type is progressive and irreversible. (Smeltzer \& Bare, 2015).

One of the complaints that can occur in patients with chronic kidney disease is uremic pruritus. It is an uncomfortable itching sensation that can reduce the quality of life of patients undergoing hemodialysis therapy. It was discovered that more than $40 \%$ of patients undergoing hemodialysis therapy experienced uremic pruritus (Nakhaee et al, 2015).

Uremic pruritus has multifactorial causes. The intensity and spatial distribution of pruritus occur very significantly over time. The mortality rates during hemodialysis therapy can increase (Combs et al., 2015) in patients with varying scales of the kidney disorder duration (Abdelghfar et al., 2017) and severe uremic pruritus.

There are many metabolic factors that have been implicated in the pathogenesis of itching, which consist of hypercalcemia, hyperphosphatemia, secondary hyperparathyroidism, and hypermagnesemia. To clarify the risk factors for the severity of uremic pruritus, the relationship between clinical and laboratory data needs to be analyzed as well as the severe tendency of uremic pruritus in a large number of patients undergoing chronic hemodialysis (Narita et al, 2006).

Based on a longitudinal study, uremic pruritus is generally reduced in patients who are about to start hemodialysis than in patients who have been on dialysis for more than 3 months who usually have moderate to severe pruritus on a scale of $42 \%$,according Stats (2017) in the United States in 2014 , as many as 118,000 people underwent chronic kidney disease treatments in both transplantation and dialysis therapies and 662,000 people underwent hemodialysis 
therapy. In Indonesia, there has been an increase in the number of patients undergoing hemodialysis therapy since 2017 (Agustiyowati, 2019). The prevalence in 2016 in Indonesia was 52,835 for patients actively undergoing therapy and 25,446 for patients experiencing early suffering. Specifically, in Haji Adam Malik General Hospital, Medan where this study was conducted, 275 patients underwent hemodialysis therapy in 2016 and 293 patients in 2017.

Some types of treatment management in patients with uremic pruritus include pharmacological, psychological, and complementary therapies (Abdelghfar, 2017). There are various kinds of essential oils used in several studies that have positive impacts on the reduction of pruritus, such as lavender, tea tree, sunflower, peppermint, jojoba essential oils, and many others.

Peppermint (mentha piperita) is one of the aromatherapy oils derived from the mint family. This plant contains essential oils of which menthol is a major component (50$60 \%$ ). It provides a cold sensation on the skin and can reduce the itching caused by histamine. The mechanism of menthol effects that can cure itching is not yet clearly known. Research findings only showed that menthol can inhibit the itching by activating A-delta fibers and K-opioid receptors (Abdelghfar, 2017).

According to Amjadi et al. (2012, peppermint also has a positive effect in reducing pruritus in pregnant women and in patients with chronic kidney failure undergoing hemodialysis therapy when applied with other essential oils (Abdelghfar et al., 2017).

Bearing in mind the impact that can be caused by uremic pruritus, researchers were interested in researching the effects of peppermint aromatherapy in reducing uremic pruritus in patients with chronic kidney disease undergoing hemodialysis.

\section{METHOD}

\section{Study Design}

This quantitative research used the quasi-experimental design with intervention and control groups. The control group of nursing care was carried out according to hospital procedures and evaluated on the scale of pruritus before and after hemodialysis.

\section{Sample and Data Collection}

The population in this study were all patients with chronic kidney disease in Haji Adam Malik General Hospital Medan, which amounted to 727 people. The research was conducted from April to May 2019. The sample amounted to 98 respondents who were counted using power analysis by using non-probability sampling with a consecutive technique. The inclusion criteria in the sampling were respondents who received hemodialysis therapy twice a week, did not experience allergies to the aromatherapy given, experienced mild to severe pruritus, did not have dermatological problems other than pruritus, were aged $18-65$ years, and did not experience open wounds in the intervention application area.

\section{Intervention}

The intervention in this study used the products of CV Subur Kimia Jaya Bandung with $100 \%$ peppermint essential oil content without a mixture of other substances as evidenced by the Refractive Index statement. The results of 1,460 were with specification values of $1.45-1,465$ in each $10 \mathrm{ml}$ container with a total of 10 black bottle containers. One to two drops were smeared topically on to the area of itching.

\section{Instrument and Data Analysis}

The itching measurement was carried out before the intervention (pretest) and after the intervention (posttest). The second week after the last intervention, the 5-D Itch Scale questionnaire was used consisting of 5 dimensions of questions that have been validated. It was found to be reliable with a CVI value of 0.90 and the reliability coefficient with Cronbach's alpha of 0.97 making the instrument feasible to use. Statistical analysis with a dependent t-test was also employed to determine the pretest-posttest effects between variables.

\section{Ethical consideration}

This study received ethical approval from the Health Research Ethics Commission of the Faculty of Nursing, Universitas Sumatera Utara (Registration No. 1668/III/SP/2019).

\section{RESULT}

The characteristics of the majority of respondents in this study were the age of late elderly (56-65 years) (with $26.5 \%$ in the intervention group and $32.7 \%$ in the control group), the majority were male $(61.2 \%$ in the intervention group and $69.4 \%$ in the control group), the duration of pruritus was more than 6 weeks with a percentage of $100 \%$ in both the intervention and control groups, the duration of hemodialysis was more than 1 year with the intervention group at $39 \%$ and the control group at73.5\%, the highest level of education was high school with the intervention group at $49 \%$ and the control group at $55.1 \%$, the employment status was unemployed with the intervention group at $73.5 \%$ and the control group at $67.3 \%$, and the comorbidity was hypertension with the intervention group at $73.5 \%$ and the control group at $71.4 \%$.

The characteristics of respondents can be seen in Table 1

Table 1. Frequency distribution and percentage of respondent characteristics

\begin{tabular}{|c|c|c|c|c|}
\hline \multirow{2}{*}{ Variable } & \multicolumn{2}{|c|}{ Intervention group } & \multicolumn{2}{|c|}{ Control group } \\
\hline & $\mathbf{n}$ & $\%$ & $\mathbf{n}$ & $\%$ \\
\hline \multicolumn{5}{|l|}{ Gender } \\
\hline Male & 30 & 61.2 & 34 & 69.4 \\
\hline Female & 19 & 38.8 & 15 & 30.6 \\
\hline \multicolumn{5}{|l|}{ Age } \\
\hline $17-25$ years old & 3 & 6.1 & 4 & 8.2 \\
\hline 26-35 years old & 7 & 14.3 & 7 & 14.3 \\
\hline $36-45$ years old & 9 & 18.4 & 10 & 20.4 \\
\hline 46-55 years old & 13 & 26.5 & 12 & 24.5 \\
\hline 56-65 years old & 13 & 26.5 & 16 & 32.7 \\
\hline \multicolumn{5}{|l|}{ Duration of pruritus } \\
\hline$>6$ weeks & 49 & 100 & 49 & 100 \\
\hline
\end{tabular}




\begin{tabular}{|c|c|c|c|c|}
\hline \multirow{2}{*}{ Variable } & \multicolumn{2}{|c|}{ Intervention group } & \multicolumn{2}{|c|}{ Control group } \\
\hline & $\mathbf{n}$ & $\%$ & $\mathbf{n}$ & $\%$ \\
\hline$<6$ weeks & 0 & 0 & 0 & 0 \\
\hline \multicolumn{5}{|l|}{ Hemodialysis period } \\
\hline 3 months & 1 & 2 & 2 & 4.1 \\
\hline 6 months & 0 & $\overline{0}$ & $\overline{1}$ & 2.0 \\
\hline 1 year & 9 & 18.4 & 10 & 20.4 \\
\hline$>1$ year & 39 & 79.6 & 36 & 73.5 \\
\hline \multicolumn{5}{|l|}{ Employment status } \\
\hline Unemployed & 36 & 73.5 & 33 & 67.3 \\
\hline Entrepreneur & 7 & 14.3 & 9 & 18.4 \\
\hline Farmer & 1 & 2.0 & 1 & 2.0 \\
\hline Government Employee & 5 & 10.2 & 6 & 12.2 \\
\hline \multicolumn{5}{|l|}{ Education } \\
\hline Elementary School & 9 & 18.4 & 27 & 55.1 \\
\hline Middle school & 7 & 14.3 & 10 & 20.4 \\
\hline High school & 24 & 49.0 & 2 & 4.1 \\
\hline College & 9 & 18.4 & 10 & 20.4 \\
\hline \multicolumn{5}{|l|}{ Disease history } \\
\hline Hypertension & 36 & 73.5 & 35 & 71.4 \\
\hline DM & 9 & 18.4 & 9 & 18.4 \\
\hline DM and gout Arthritis & 1 & 2 & 0 & 0 \\
\hline Gout and arthritis & 2 & 4.1 & 2 & 6.1 \\
\hline DM and hypertension & 1 & 2 & 3 & 4.1 \\
\hline
\end{tabular}

Table 2. Analysis before giving peppermint aromatherapy in chronic kidney failure patients who underwent hemodialysis therapy $(n=49)$

\begin{tabular}{lcccccccc} 
& \multicolumn{3}{c}{ Intervention Group } & \multicolumn{3}{c}{ Control group } \\
\cline { 2 - 9 } Degree of Pruritus & Pre-Intervention & Post Intervention & \multicolumn{2}{c}{ Pre-Control } & \multicolumn{3}{c}{ Post Control } \\
\cline { 2 - 9 } & $\mathbf{n}$ & $\%$ & $\mathbf{n}$ & $\%$ & $\mathbf{n}$ & $\%$ & $\mathbf{n}$ & $\%$ \\
\hline Mild & 12 & 24.5 & 25 & 51.0 & 13 & 15.9 & 8 & 9.8 \\
Moderate & 29 & 59,2 & 24 & 49.0 & 28 & 34.1 & 34 & 41.5 \\
Severe & 8 & 16.3 & 0 & 0 & 8 & 9.8 & 7 & 8.5 \\
\hline
\end{tabular}

Based on Table 2, the majority of respondents experienced uremic pruritus of a moderate intensity, both in the intervention group (59.2\%) and in the control group (34.1\%) before the intervention. Meanwhile, the majority of respondents experienced a decrease on the uremic pruritus scale after the intervention, which was within the limit of mild intensity $(51.0 \%)$ but it was moderate in the control group $(41.5 \%)$.

Table 3. Comparison in the degree of uremic pruritus between the intervention and control groups

\begin{tabular}{lccccc}
\hline \multirow{2}{*}{ Group } & \multicolumn{2}{c}{ Pre-test } & \multicolumn{2}{c}{ Post-Test } & \multirow{2}{*}{ P } \\
\cline { 2 - 5 } & $\mathbf{M} \pm$ SD & Min-max & M \pm SD & Min-Max & 0.000 \\
\hline Intervention group & $25.0 \pm 14.2$ & $5-30$ & $1.49 \pm 0.50$ & $5-20$ & 0.102 \\
Control group & $1.90 \pm 0.65$ & $6-28$ & $1.98 \pm 0.55$ & $7-30$ & \\
\hline
\end{tabular}

Based on Table 3 and by using the Wilcoxon test, it resulted in a $p$ value of 0.000 for the intervention group and a $p$ value of 0.102 for the control group. There is an effect of peppermint aromatherapy in reducing uremic pruritus in patients with chronic renal failure undergoing hemodialysis therapy.

\section{DISCUSSION}

Before the intervention, the results showed that the intensity of uremic pruritus experienced by the majority of respondents was moderate with a hemodialysis frequency of twice a week. Providing adequate and controlled hemodialysis measures can reduce uremia and uremic pruritus. Therefore, it was found that respondents who regularly receive hemodialysis experienced a lower incidence of uremic pruritus (Mathur et al., 2010).

Germain (2017) stated that more than $40 \%$ of patients with chronic kidney disease (end-stage) who underwent hemodialysis experienced uremic pruritus starting from moderate to severe intensity.

Hemodialysis that has been carried out for a longer period can increase the incidence of uremic pruritus (Germain, 2017; Lin et al., 2012). Following the results of the study, it was found that the majority of respondents who experienced uremic pruritus were respondents who had spent years undergoing hemodialysis (more than one year). However, this study is not in line with the results of research conducted by Tarp (2017). It was discovered that more patients experienced uremic pruritus in the first 3 months after hemodialysis and even 6 to 12 months in a row after hemodialysis.

According to Narita et al. (2006), the causes of uremic pruritus are not yet clearly known but there are several risk factors (i.e. it is multifactorial). In Kimata et al.'s (2014) study, the latest theory identified that uremic pruritus is associated with a decrease in the immune system. 
In this study, the age of the early elderly (46-55 years) and late elderly (56-65 years) constitute the majority of those suffering from uremic pruritus. This can be attributed to the elderly age in which there is a decrease in bodily functioning, specifically a decrease in endurance. Consequently, elderly people are more prone to uremic pruritus. Another study that had the same results as this study was conducted by Berger \& Steinhoff (2011), which stated that elderly age has a relationship with the tendency or severity of uremic pruritus in chronic kidney disease respondents. However, it does not have the same results as a study conducted by Szepietowski et al. (2002), which states that age does not affect the occurrence of uremic pruritus.

In several studies, it was found that the gender factor also influences the occurrence of uremic pruritus. In this study, male respondents experience more uremic pruritus than women. In this case, gender is associated with the pathogenesis of uremic pruritus (Berger \& Steinhoff, 2011; Lin et al. 2012; Combs et al. 2015; Aval et al. 2018).

Another factor that can aggravate the occurrence of uremic pruritus is having comorbidities such as hypertension and diabetes mellitus (Kimata et al., 2014). In this study, the majority of respondents experienced the comorbidities of hypertension and diabetes mellitus. This can occur where the comorbidity itself or drugs consumed can trigger uremic pruritus (Mettang et al., 2015; Nakamoto et al., .2019). Nonetheless, Narita et al. (2006) found that comorbidities such as diabetes and hypertension are not independent factors that can aggravate the occurrence of uremic pruritus.

The impact of uremic pruritus on the majority of respondents experiencing sleep disturbance was measured through the 5-D Itch scale questionnaire consisting of five question domains along with the distribution of the itch felt by the respondent. Patients experiencing difficulty sleeping and sometimes waking up at night due to pruritus was at $46.9 \%$. This is in line with Mathur's (2010) and Combs et al.'s (2015) statements who found that patients who experience uremic pruritus will experience sleep disorders.

Research conducted by Kimball et al. (2016) stated that in general, uremic pruritus can interfere with sleep. As a result, it inhibits or interferes with work activities due to the lack of sleep. In this study, the majority of respondents who did not work was $51.3 \%$. This study is in line with the research of Lin et al. (2012) who found that uremic pruritus can affect social activities and work productivity.

Another statement in the study of Simonsen et al. (2017) was that respondents who experience uremic pruritus also experience an interference with social activities and quality of life. Therefore, it can increase mortality.

The duration of uremic pruritus experienced by respondents in the study comprises $49 \%$ with a duration of $<6$ hours/day over the last 2 weeks, while $4.1 \%$ had the highest duration of 18-23 hours/day. The intensity of uremic pruritus felt by the majority of respondents was moderate. The tendency of uremic pruritus felt by the majority of respondents $(44.9 \%)$ was that it was getting worse over the last two weeks. This is attributed to the tendency of uremic pruritus to fluctuate over time. The stable pruritus will arise episodically because it occurs throughout the bloodstream or it is systemic due to urea buildups. This occurs because the kidneys are unable to filter, clean the blood of foreign substances that enter the body through daily consumption of food and drinks, and excrete maximally. This is in line with what is stated by Combs et al. (2015) and Berger and Steinhoff (2011) that uremic pruritus is felt by the respondents almost every day intermittently or sometimes it is on and off.

The distribution of pruritus varies but the most commonly experienced by respondents in this study is in the area of the back with the results obtained as much as $77.6 \%$. But, the areas of the buttocks and abdomen are also frequent problem areas. The back area is the most common because it is the most extensive and often stressed. It has the same results as found by Berger and Steinhoff (2011), Simonsen et al. (2017), and Minato, et al. (2020) that the most common site of pruritus is on the back.

After the intervention, the pruritus intensity experienced by respondents was mild (6-14). Abdelghfar et al. (2017) stated that providing a complementary intervention such as essential oils, especially the one containing menthol, can alleviate the uremic pruritus felt by respondents. Another study presented by Elsaie et al. (2016) affirms that peppermint essential oil can reduce pruritus not only in patients with chronic kidney disease but also in patients with diabetes mellitus and liver disorders by giving it for two weeks. In Amjadi's research (2012), it is also stated that pruritus in pregnant women can decrease by giving peppermint essential oil through inhalation.

The uremic pruritus felt by respondents had decreased but the duration experienced by the majority took place in less than 6 hours. The tendency of pruritus that did not interfere with sleep was $61.2 \%$. Nevertheless, the distribution of pruritus remains dominant in the respondent's back, abdomen, and extremities. This is due to the uremic pruritus occurring in one-third of the respondent's body area even after the intervention. It will occur episodically to a mild intensity if the effects of peppermint essential oil have begun to disappear or diminish.

Several complementary intervention studies that have been carried out to reduce the scale of pruritus due to kidney failure, diabetes, or liver disease have given good results. Various oils such as lavender essential oil, baby oil, and peppermint essential oil have had a positive impact on patients with uremic pruritus in respondents experiencing chronic kidney disease. This is in line with research conducted by Abdelghfar et al. (2017) and Elsaie et al. (2016).

Aromatherapy is a common complementary and alternative treatment modality, which involves herbal medicines that utilize various oils extracted from plants and trees that can be given through skin absorption or inhalation (Mapp and Hostetler, 2020).

Based on the results of this study, there are effects of peppermint aromatherapy interventions in reducing uremic pruritus in patients with chronic kidney disease undergoing hemodialysis therapy through the skin or topical medication.

Orchard and Vuuren (2017) state that essential oils are one of the most popular natural products, with one of the main applications being skin medication. Combs et al. (2015) stated that one of the therapies used as a treatment for reducing uremic pruritus is a topical treatment because it can also improve skin hydration, where one of the factors that cause uremic pruritus is a dry skin condition. 
The peppermint essential oil has effects in reducing the scale of uremic pruritus. This is due to the peppermint content that has menthol, which can provide a cold sensation on the skin that can work as an antihistamine. Moreover, its major component is menthol in a concentration of $50-60 \%$ (Abdelghfar et al., 2017).

The nursing theory used in this research is Kolcaba's Comfort Theory (2001) where the roles of nurses comprise providing patient comfort physically, psychologically, spiritually, and socially. The comforting context can be seen through physical, psycho-spiritual, environmental, and social values from the 3 ideas put forward by Kolcaba (2001) namely relief, ease (tranquility), and transcendence (helping patients in trouble) (Alligood, 2014). This is in line with the results of research showing peppermint aromatherapy can reduce the pruritus scale and provide comfort by using the $5-D$ Itch Scale questionnaire measurement tool. The scale consists of 5 categories of questions namely duration (how long the pruritus has been felt for), intensity (how severe the pruritus is), the tendency (whether the pruritus is getting better or worse), impact (on sleep, social, personal, and work activities), and distribution (spread of pruritus to other parts of the body). The limitation of this research is that it does not control the laboratory values associated with elevated uremic levels.

\section{CONCLUSION AND RECOMMENDATION}

Peppermint essential oil complementary therapy has positive effects on patients with chronic kidney disease who experience uremic pruritus. Peppermint aromatherapy is a safe therapy that is applied topically and can reduce the severity of pruritus. The implication of this research is that providing atopical complementary therapy with peppermint essential oilcan reduce the scale of uremic pruritus in addition to pharmacological (antipruritic) medication.

\section{REFERENCES}

Abdelghfar, S. Z., Elsebae, H. A., Elhadry, S. M., \& Hassan, A. A. Effect of aromatherapy on uremic pruritus among patients undergoing hemodialysis. (2017). IOSR Journal of Nursing and Health Science (IOSRJNHS e-ISSN: 2320-1959.p- ISSN: 2320-1940 Volume 6, Issue 2 Ver. VIII (Mar. - Apr. 2017), PP 22-30 www.iosrjournals.org

Agustiyowati, T. H. R. (2019). The Influence of Hemodialysis Adequacy on the Blood Urea Value. KnE Life Sciences, 152-157.

Alligood, M. R., \& Tomey, A. M. (2014). Nursing Theorists and Their Work. St Louis, MO.

Amjadi, M. A., Mojab, F., \& Kamranpour, S. B. (2012). The effect of peppermint oil on symptomatic treatment of pruritus in pregnant women. Iranian journal of pharmaceutical research: IJPR, 11(4), 1073.

Aval, S. B., Ravanshad, Y., Azarfar, A., Mehrad-Majd, H., Torabi, S., \& Ravanshad, S. (2018). A Systematic Review and Meta-analysis of Using Acupuncture and Acupressure for Uremic Pruritus. Iranian journal of kidney diseases, 12(2), 78. handbook for practice, 637-659.

Berger, T. G., \& Steinhoff, M. (2011, June). Pruritus and renal failure. In Seminars in cutaneous medicine and surgery (Vol. 30, No. 2, p. 99). NIH Public Access.

Combs, S. A., Teixeira, J. P., \& Germain, M. J. (2015, July). Pruritus in kidney disease. In Seminars in nephrology (Vol. 35, No. 4, pp. 383-391). WB Saunders.

Elsaie, L. T., El Mohsen, A. M., Ibrahim, I. M., Mohey-Eddin, M. H., \& Elsaie, M. L. (2016). Effectiveness of topical peppermint oil on symptomatic treatment of chronic pruritus. Clinical, cosmetic and investigational dermatology, 9, 333.

Germain, M. J. (2017). Uremic pruritus: an itch with ominous consequences. American nephrology, 46(6), 448-450.

journal of

Kimata, N., Fuller, D. S., Saito, A., Akizawa, T., Fukuhara, S., Pisoni, R. L., ...\& Akiba, T. (2014). Pruritus in hemodialysis patients: Results from the Japanese Dialysis Outcomes and Practice Patterns Study (JDOPPS). Hemodialysis International, 18(3), 657667.

Kimball, A. B., Edson-Heredia, E., Zhu, B., Guo, J., MaedaChubachi, T., Shen, W., \& Bianchi, M. T. (2016). Understanding the Relationship Between Pruritus Severity and Work Productivity in Patients with Moderate-to-Severe Psoriasis: Sleep Problems Are a Mediating Factor. Journal of drugs in dermatology: $J D D, 15(2)$, 183-188.

Kolcaba, K. (2001). Evolution of the mid-range theory of comfort for outcomes research. Nursing outlook, 49(2), 86-92.

Lin, T. C., Lai, Y. H., Guo, S. E., Liu, C. F., Tsai, J. C., Guo, H. R., \& Hsu, H. T. (2012). Baby oil therapy for uremic pruritus in hemodialysis patients. Journal of clinical nursing, 21(12), 139-148

Mapp, C. P., Hostetler, D., Sable, J. F., Parker, C., Gouge, E., Masterson, M., ... \& Higgins, M. (2020). Peppermint Oil: Evaluating Efficacy on Nausea in Patients Receiving Chemotherapy in the Ambulatory Setting. Clinical journal of oncology nursing, 24(2), 160-164.

Mathur, V. S., Lindberg, J., Germain, M., Block, G., Tumlin, J., Smith, M., \& ITCH National Registry Investigators. (2010). A longitudinal study of uremic pruritus in hemodialysis patients. Clinical Journal of the American Society of Nephrology, 5(8), 1410-1419

Mettang, T., \& Kremer, A. E. (2015). Uremic pruritus. Kidney International, 87 (84), 685-691.

Minato, S., Hirai, K., Morino, J., Kaneko, S., Yanai, K., Mutsuyoshi, Y., ... \& Aomatsu, A. (2020). Factors Associated with Uremic Pruritus in Patients Undergoing Peritoneal Dialysis. International Journal of Nephrology and Renovascular Disease, 13, 1.

Nakamoto, H., Kobayashi, T., Noguchi, T., Kusano, T., Ashitani, K., Imaeda, H., \& Maezono, M. (2019). Prevalence and Severity of Itching in Patients with End-Stage Renal Disease: Treatment with Nalfurafine Hydrochloride. Blood purification, 47(2), 45-49.

Nakhaee, S., Nasiri, A., Waghei, Y., \& Morshedi, J. (2015). Comparison of Avena sativa, vinegar, and hydroxyzine for uremic pruritus of hemodialysis patients: a crossover randomized clinical trial. Iranian journal of kidney diseases, 9(4), 316.

Narita, I., Alchi, B., Omori, K., Sato, F., Ajiro, J., Saga, D., ...\& Akazawa, K. (2006). Etiology and prognostic significance of severe uremic pruritus in chronic 
hemodialysis patients. Kidney international, 69(9), 1626-1632.

Orchard, A., Sandasi, M., Kamatou, G., Viljoen, A., \& van Vuuren, S. (2017). The in vitro antimicrobial activity and chemometric modelling of 59 commercial essential oils against pathogens of dermatological relevance. Chemistry \& biodiversity, 14(1), e1600218.

Simonsen, E., Komenda, P., Lerner, B., Askin, N., Bohm, C., Shaw, J., ...\& Rigatto, C. (2017). Treatment of uremic pruritus: a systematic review. American Journal of Kidney Diseases, 70(5), 638-655.
Smeltzer, S. C. \& Bare (2015). Brunner \& Suddarth's Textbook of Medical Surgical nursing. Philadelpia: Lippincott.

Stats, F. National Chronic Kidney Disease Fact Sheet. (2017). Prevalence Chronic Kidney Diseases

Szepietowski, J. C., Sikora, M., Kusztal, M., Salomon, J., Magott, M., \& Szepietowski, T. (2002). Uremic pruritus: a clinical study of maintenance hemodialysis patients. The Journal of dermatology, 29(10), 621627.

Tarp, H., BondePetersen, M., \& Finderup, J. (2017). Patients in Haemodialysis Experienced Uremic Pruritus as a Dual Phenomenon. Journal of renal care, 43(1), 21-28. 